\title{
Diffusion tensor imaging assessment of microstructural brainstem integrity in Chiari malformation Type I
}

\author{
Vibhor Krishna, MD, ScM, ${ }^{1,2}$ Francesco Sammartino, MD, ${ }^{1}$ Philip Yee, BSc, ${ }^{1}$ David Mikulis, MD, ${ }^{1,3,4-6}$ \\ Matthew Walker, MSc, ${ }^{3}$ Gavin Elias, BA, ${ }^{1}$ and Mojgan Hodaie, MD, MSc ${ }^{1,3-5}$

\begin{abstract}
${ }^{1}$ Division of Neurosurgery, Department of Surgery, ${ }^{4}$ Institute of Medical Science, and ${ }^{6}$ Department of Medical Imaging, University of Toronto; ${ }^{3}$ Division of Brain Imaging, Behaviour Systems Neuroscience, Toronto Western Research Institute; and ${ }^{5} \mathrm{~J}$ oint Department of Medical Imaging, University Health Network, Toronto, Ontario, Canada; and ${ }^{2}$ Center for Neuromodulation,
\end{abstract} \\ Department of Neurosurgery, Ohio State University, Columbus, Ohio
}

\begin{abstract}
OBJECTIVE The diagnosis of Chiari malformation Type I (CM-I) is primarily based on the degree of cerebellar tonsillar herniation even though it does not always correlate with symptoms. Neurological dysfunction in CM-I presumably results from brainstem compression. With the premise that conventional MRI does not reveal brain microstructural changes, this study examined both structural and microstructural neuroimaging metrics to distinguish patients with CM-I from age- and sex-matched healthy control subjects.
\end{abstract}

METHODS Eight patients with CM-I and 16 controls were analyzed. Image postprocessing involved coregistration of anatomical T1-weighted with diffusion tensor images using 3D Slicer software. The structural parameters included volumes of the posterior fossa, fourth ventricle, and tentorial angle. Fractional anisotropy (FA) was calculated separately in the anterior and posterior compartments of the lower brainstem.

RESULTS The mean age of patients in the CM-I cohort was $42.6 \pm 10.4$ years with mean tonsillar herniation of $12 \mathrm{~mm}$ $(S D 0.7 \mathrm{~mm})$. There were no significant differences in the posterior fossa volume $(p=0.06)$ or fourth ventricular volume between the 2 groups $(p=0.11)$. However, the FA in the anterior brainstem compartment was significantly higher in patients with CM-I preoperatively ( $p=0.001$ ). The FA values normalized after Chiari decompression except for persistently elevated FA in the posterior brainstem compartment in patients with $\mathrm{CM}-\mathrm{I}$ and syrinx.

CONCLUSIONS In this case-control study, microstructural alterations appear to be reliably associated with the diagnosis of CM-I, with a significantly elevated FA in the lower brainstem in patients with CM-I compared with controls. More importantly, the FA values normalized after decompressive surgery. These findings should be validated in future studies to determine the significance of diffusion tensor imaging-based assessment of brainstem microstructural integrity as an adjunct to the clinical assessment in patients with CM-I.

http://thejns.org/doi/abs/10.3171/2015.9.JNS151196

KEY WORDS Chiari malformation; imaging marker; brainstem compression; diffusion tensor imaging; diffusion-weighted imaging; fractional anisotropy; anatomy

$\mathrm{C}$ HIARI malformation Type I (CM-I) typically presents with suboccipital headache worsened with Valsalva maneuvers. ${ }^{8,17}$ Besides pain, patients also experience symptoms related to brainstem compression, e.g., dysphagia, dizziness, sensory dysfunction, and motor weakness. ${ }^{4,17,24}$ Conventionally, the imaging diagnosis of $\mathrm{CM}-\mathrm{I}$ is based on the degree of cerebellar herniation rather than severity of brainstem compression. ${ }^{17,22,29}$ However, a significant proportion of individuals with tonsillar herniation are asymptomatic. ${ }^{16,25}$ The clinical correlation of other structural parameters, including posterior fossa volume or the ratio of posterior fossa volume to the supratentorial volume, is also poor. ${ }^{16,25,27}$ For example, smaller posterior fossa volumes are not associated with a diagnosis of CM-I. ${ }^{27}$ Therefore, novel imaging assessment methods associated with the severity of brainstem compression are needed.

Diffusion tensor imaging (DTI) has primarily been investigated for studying the structural connectivity in the CNS. ${ }^{19}$ Additionally, the quantitative assessment of diffusion restriction is a marker of the neuronal microstructural integrity. ${ }^{13}$ Fractional anisotropy (FA) is a composite mea-

ABBREVIATIONS AC = anterior commissure; $\mathrm{CM}-\mathrm{I}=$ Chiari malformation Type I; DTI = diffusion tensor imaging; $\mathrm{FA}=$ fractional anisotropy; $\mathrm{PC}=$ posterior commissure; $\mathrm{ROI}=$ region of interest.

SUBMITTED May 26, 2015. ACCEPTED September 15, 2015.

INCLUDE WHEN CITING Published online February 5, 2016; DOI: 10.3171/2015.9.JNS151196. 
sure of magnitude and directionality of diffusion restriction. FA can vary between 0 and 1 ; the white matter is typically more than 0.2 , whereas the gray matter is less than 0.2. ${ }^{19}$ An external compression on the brain would initially increase anisotropy due to diffusion restriction in the direction perpendicular to the compression. However, prolonged or severe compression may cause irreversible injury (e.g., edema, hemorrhage, demyelination, or axonal loss) and an unrestricted diffusion of water molecules, resulting in a decline in FA. ${ }^{13,14,20}$ In CNS locations with densely packed white matter tracts (e.g., spinal cord and lower brainstem), FA changes can also be detected in regions immediately proximal to the site of compression. ${ }^{9,11}$ Therefore, the FA in the brainstem should theoretically be elevated immediately proximal to tonsillar herniation in patients with CM-1. If this hypothesis is correct, then the presumed FA elevation should normalize after decompressive surgery. To test this hypothesis, we compared a cohort of patients with CM-I with healthy control subjects. The FA values were calculated separately in the anterior and posterior compartments of the brainstem due to their distinct functional neuroanatomy. ${ }^{2}$

\section{Methods}

This study was approved by the institutional ethics board at the University Health Network at the University of Toronto.

\section{Study Subjects and Imaging Protocol}

All consecutive patients with CM-I (20 patients) who underwent decompression between 2008 and 2014 at our center were reviewed. Patients who underwent pre- and postoperative 3-T (GE Signa) DTI extending to the lower brainstem were included. Six patients with missing images (either pre- or postoperative), and another 6 patients with images that had significant motion artifact or missing or incomplete image sets were excluded. Eight patients with CM-I with complete preoperative imaging data were included for further clinical and imaging analysis. One patient with CM-I (Case 8) did not undergo postoperative DTI.

The DT protocol included 60 directions of diffusion gradients $\left(b=1000 \mathrm{sec} / \mathrm{mm}^{2} ; 0.94 \times 0.94 \times 3-\mathrm{mm}\right.$ voxel size; TE $86.6 \mathrm{msec}$; TR $12,000 \mathrm{msec}$; and $128 \times 128 \mathrm{ma}-$ trix). Structural 3D fast spoiled gradient echo axial T1weighted images were also acquired $(0.85 \times 0.85 \times 1-\mathrm{mm}$ voxel size; $256 \times 256$ matrix; FOV $220 \mathrm{~mm}$; TE $5 \mathrm{msec}$; TR $12 \mathrm{msec}$; and TI $300 \mathrm{msec}$ ). Two age- and sex-matched controls were identified for each patient with CM-I from our imaging database of healthy subjects scanned using the same scanner and with the same imaging protocol. All of the patients with CM-I underwent posterior fossa decompression with duroplasty, using autologous nuchal fascia graft similar to the technique described elsewhere. ${ }^{28}$

\section{Imaging Analysis: Calculation of Structural Parameters and FA Values}

The imaging analysis was performed using 3D Slicer software by investigators blinded to the clinical outcomes (V.K., F.S., P.Y., and M.W.). Images were postprocessed and the corrections for motion and eddy current artifacts (using GTRACT extension) were applied. The DT images were coregistered by first registering the diffusion-weighted images to the B0 image (using affine transformation) and then coregistering the anatomical T1-weighted images with the B0 image (using rigid-body registration). The accuracy of coregistration was also visually confirmed. Finally, whole-brain tensor calculation was performed.

Among the structural parameters, we included tonsillar herniation, posterior fossa volume, and total brain volume because these can have significant alteration in patients with CM-I. ${ }^{17,22,25,29}$ In addition, we calculated the volume of the fourth ventricle, intercommissural distance, and tentorial angle. The images were first aligned to the plane of anterior and posterior commissures (AC and PC). The tonsillar herniation was calculated from the tip of the ectopic cerebellar tonsil and McRae's line, as described elsewhere. ${ }^{16}$

For calculation of the posterior fossa volume, we generated the label maps on the sagittal projections extending from the inferior surface of the tentorium to the foramen magnum. To distinguish the supratentorial from the infratentorial fossa in the parasagittal projections, closer to midline, we drew a perpendicular line from the tentorial edge to the clivus.

The total brain volume was calculated using a 3D Slicer toolbox. We manually created label maps to calculate the volume of the fourth ventricle separately in the superior and inferior compartments (demarcated by the intersection of superior and inferior medullary velum). The top of the fourth ventricle was identified in the axial projections at the pontomedullary junction and the bottom edge was chosen at the transition between medulla and spinal cord. The lateral and midline medullary cisterns (extending toward the foramen of Luschka and Magendie, respectively) were excluded from the label map. The intercommissural distance (AC-PC length) was calculated in the axial images from the posterior edge of the $\mathrm{AC}$ to the anterior edge of the PC. Finally, the tentorial angle was calculated in the midsagittal projections from a horizontal line (parallel to the intercommissural plane) joining the base of the tentorium with the clivus.

For calculation of FA, we first identified T1-weighted axial projections at the level of the foramen magnum, the presumed site of maximum brainstem compression. Subsequently, in the coregistered FA images, we selected 2 adjacent axial slices (3-mm slice thickness each) immediately proximal to the slice with maximum compression. These slices were uniformly selected and used for FA calculation across the patients with CM-I and controls. As shown in Fig. 1, we created 2 label maps in these 2 axial slices to distinguish the anterior and posterior compartments of the lower brainstem, demarcated by the retroolivary sulcus. The mean FA values were separately calculated for the anterior and posterior compartments of the brainstem due to their inherently distinct neuroanatomy. ${ }^{2}$

\section{Clinical Information}

Standardized data abstraction sheets were created to retrospectively extract clinical information from patient charts. The clinical outcomes were assessed by a blinded 

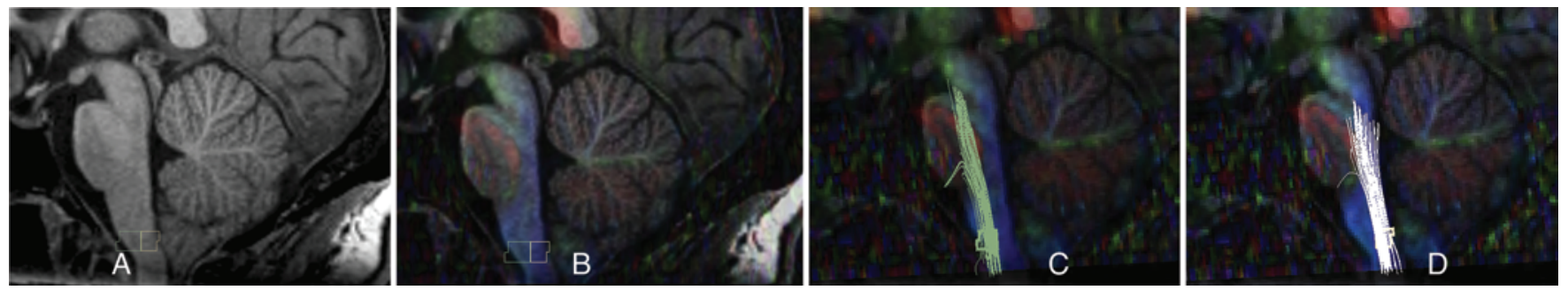

FIG. 1. The methodology for creating the label maps is shown in a sagittal T1-weighted image (A) and the directionally encoded color map coregistered with the T1-weighted image (mixed color by orientation/anatomical views) (B). The area of lower brainstem immediately proximal to the foramen magnum (the presumed site of maximum compression) was identified. Label maps were then created separately for the anterior (green) and posterior (white) brainstem compartments. These label maps were checked for anatomical accuracy with the structural T1-weighted scans in both axial and sagittal planes. The white matter tracts traversing the anterior (C) and posterior (D) compartments are separately visualized. Anatomically, the anterior label map includes the pyramidal tract, medial lemniscus, and central tegmental tract; the posterior label map includes the medial longitudinal fasciculus, inferior cerebellar peduncle, spinothalamic tract, and spinal trigeminal tract. Figure is available in color online only.

rater (G.E.). We incorporated information regarding demographic data (i.e., age and sex); Chiari-related symptoms (all symptoms, duration, and associated characteristics); neurological examination findings (lower cranial nerve dysfunction, swallow study findings, myelopathy, and sensory abnormalities); presence of Chiari-associated conditions (syrinx, hydrocephalus); operative course (any complications, additional procedures for Chiari-associated conditions); and postoperative details. The severity of CM-I was assessed with the Chiari severity score. ${ }^{12}$ Based on this scale, patients with CM-I are classified into 3 groups of increasing severity (Grades 1-3) after considering both the clinical (headache, myelopathy) and radiographic (tonsillar herniation, location and extent of syrinx) findings. The outcomes were assessed using the Chiari outcomes scale. ${ }^{3}$ This scale assesses the recovery in 4 major domains (pain resolution, non-pain-symptom resolution, disability, and complications), each on a 4-point scale.

\section{Statistical Analysis}

We used SPSS (v. 22; IBM Corp.) for statistical analysis. Continuous variables were summarized as the mean and SDs, and the categorical variables as proportions. The normal distribution of data was confirmed using the Shapiro-Wilk test. We used the t-test and ANOVA to compare the continuous variables and the chi-square test to compare the categorical variables.

\section{Results}

\section{Description of the Study Cohorts}

The individual clinical characteristics of patients with CM-I are shown in Table 1. The mean age of this cohort was $42.6 \pm 10.4$ years and the majority ( 7 of 8 patients) were women. All of the patients presented with typical headaches (suboccipital pain worsened with Valsalva maneuvers) and a variety of symptoms related to brainstem compression, e.g., dizziness, myelopathy, absent gag reflex, and sensory deficits. The duration of symptoms ranged from 6 months to 13 years. Three patients were found to have preoperative syrinx (Cases 2, 4, and 6) that resolved after surgery without additional syrinx-related procedures. The decompressive surgery was uneventful, with no complications in any patient. The mean follow-up of this cohort was $14.3 \pm 10.9$ months. The Chiari outcomes scores ranged from 9 to 15 .

Sixteen age- and sex-matched controls were selected from the imaging database for comparison with patients with CM-I. The mean age of this cohort was $42.3 \pm 10.3$ years.

\section{Comparison of Structural Parameters Between Patients With CM-I and Controls}

None of the structural parameters (i.e., intercommissural distance, posterior fossa volume, fourth ventricular volume, and total brain volume) were significantly different between patients with CM-I and controls (Table 2). There was a trend toward smaller posterior fossa volume in patients with CM-I $(\mathrm{p}=0.06)$.

\section{The FA Value Was Significantly Elevated in Patients With CM-I}

In the anterior brainstem compartment, the mean FA for patients with CM-I was $0.52 \pm 0.09$ compared with $0.42 \pm 0.05$ for controls (1-way ANOVA, $p=0.001$ ). There was a trend toward a higher mean FA in the posterior compartment $(0.52 \pm 0.08$ in patients with CM-I vs $0.48 \pm 0.05$ in controls; $p=0.09$ ), although this difference was not statistically significant. The scatterplots and mean FA values comparing patients with CM-I with controls are shown in Fig. 2. In 6 of 8 patients with CM-I, the FA values in the anterior brainstem were higher than in both matched controls. However, in $2(25 \%)$ patients, the FA values in the anterior brainstem were either similar to or lower than those of their matched controls.

\section{The FA Value Normalized After Decompressive Surgery}

The FA value decreased to $0.47 \pm 0.16$ in the anterior and $0.45 \pm 0.15$ in the posterior brainstem compartment after decompression. These postoperative FA values were not significantly different from those of the controls (1way ANOVA, $p=0.24$ and $p=0.53$, respectively).

\section{Predictors of Postoperative Changes in FA Values}

In patients with CM-I and syrinx, the FA values were persistently elevated in the posterior brainstem compartment. The postoperative FA increased by $13.4 \%$ (SD 


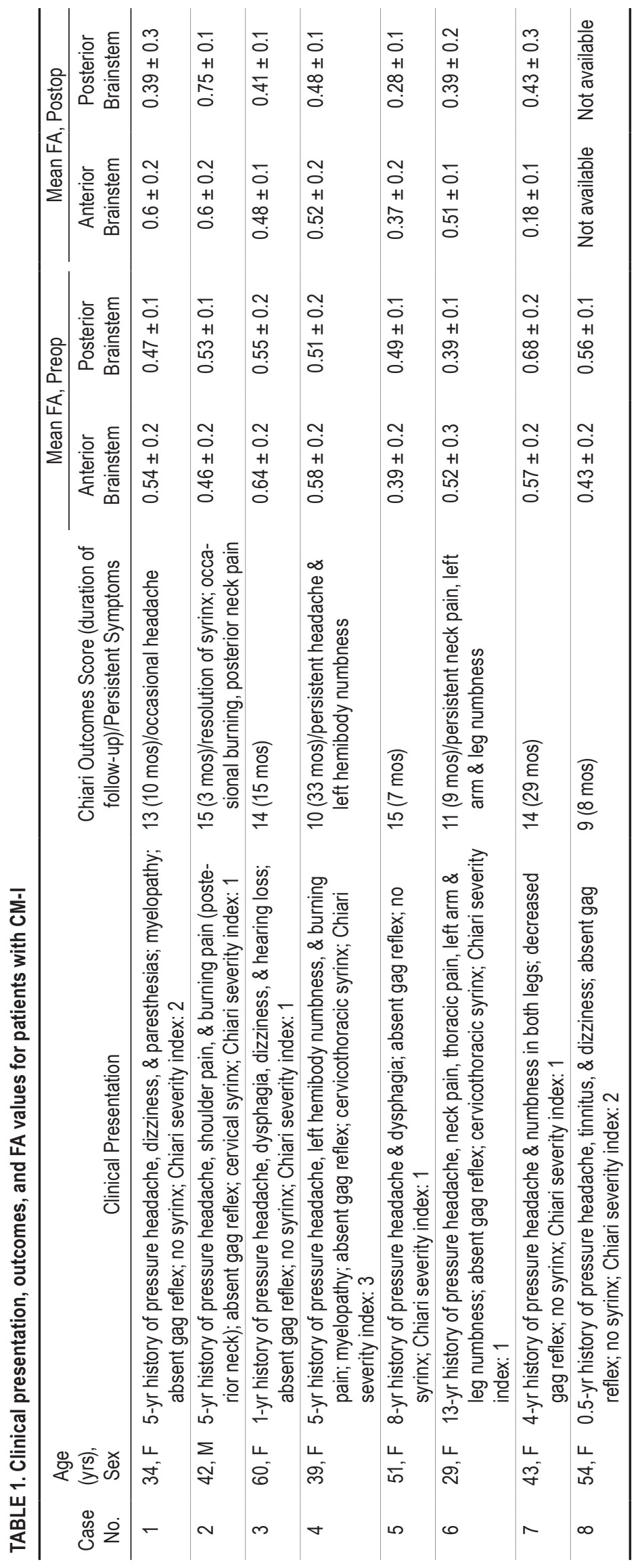


TABLE 2. Comparison of demographic data and structural imaging parameters between patients with $\mathrm{CM}-\mathrm{I}$ and healthy control subjects

\begin{tabular}{lccc}
\hline \multicolumn{1}{c}{ Variable } & Patients w/ CM-I, $\mathrm{n}=8$ & Controls, $\mathrm{n}=16$ & $\mathrm{p}$ Value \\
\hline Tonsillar herniation, $\mathrm{mm} \pm$ SD (range) & $8.8 \pm 2.6(4.7-12.5)$ & $0.44 \pm 1.1$ & 0.00001 \\
\hline AC-PC length, $\mathrm{mm}$ & $26.7 \pm 1.5$ & $25.7 \pm 1.3$ & 0.11 \\
\hline Total brain vol, $\mathrm{mm}^{3}$ & $1082.9 \pm 71.1$ & $1047.8 \pm 90.8$ & 0.36 \\
\hline Posterior fossa vol, $\mathrm{mm}^{3}$ & $153.1 \pm 14.5$ & $167.8 \pm 17.5$ & 0.06 \\
\hline Vol of fourth ventricle, $\mathrm{mm}^{3}$ & & & 0.39 \\
Total & $9.8 \pm 5.1$ & $11.4 \pm 35.6$ & 0.98 \\
$\quad$ Superior compartment & $2.9 \pm 1.7$ & $2.9 \pm 0.9$ & 0.26 \\
$\quad$ Inferior compartment & $6.8 \pm 3.7$ & $8.4 \pm 2.9$ & 0.78 \\
\hline Tentorial angle & $56 \pm 6.5$ & $56.7 \pm 5.1$ & \\
\hline
\end{tabular}

26.5\%) in the posterior brainstem compartment in patients with syrinx in contrast to a $30.9 \%$ (SD 10.9\%) decline in patients without syrinx (1-way ANOVA, $p=0.027$ ). A similar trend, although not statistically significant, was observed in the anterior brainstem compartment (a decline of $22 \% \pm 34.7 \%$ in patients with CM-I without syrinx vs an elevation of $8.7 \% \pm 25.4 \%$ in patients with syrinx; 1-way ANOVA, $\mathrm{p}=0.26$ ).

The individual pre- and postoperative FA values are plotted in an area diagram in Fig. 3. The blue area denotes a higher preoperative FA value, whereas red denotes a higher postoperative FA. Patients with CM-I and syrinx (Cases 2, 4, and 6) had persistent significant elevation of FA in the posterior brainstem compartment.

\section{Discussion}

This study aimed to find better neuroimaging correlates of CM-I by comparing both structural and microstructural parameters in a cohort of patients with CM-I with age-matched controls, to determine which parameters best correlate with clinical findings. Among the structural parameters, we included previously studied measurements (tonsillar herniation, posterior fossa volume, and total brain volume) and novel measures such as volume of the fourth ventricle, its compartments, and the tentorial angle. Microstructural measures, on the other hand, were based primarily on FA metrics of the anterior and posterior brainstem; these are metrics that are diffusion based and cannot be readily obtained from conventional MRI.
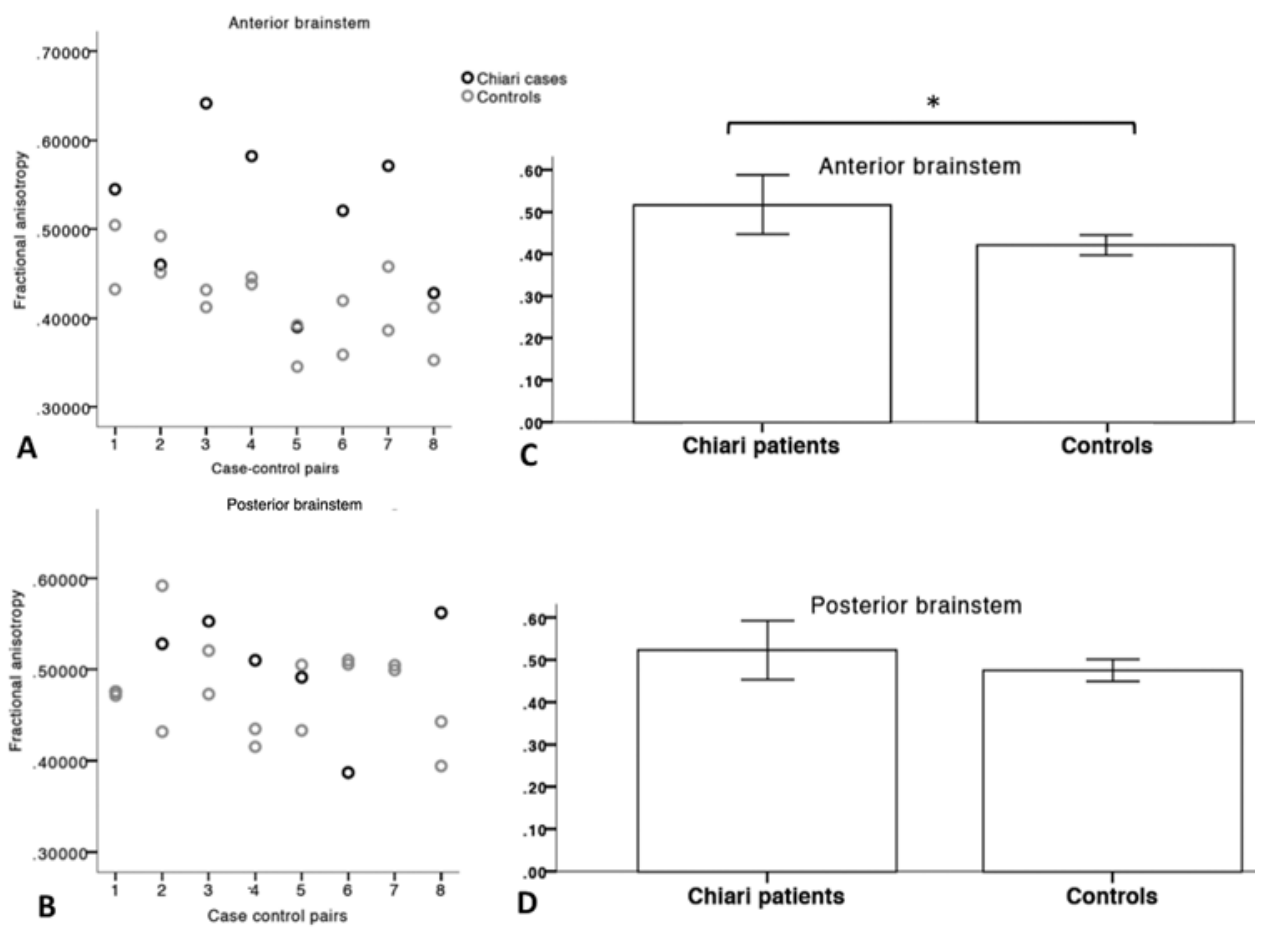

FIG. 2. The FA values in the anterior and posterior brainstem compartments. A: Scatterplot of FA values in the anterior brainstem compartment. Patients with CM-I (dark circles) and corresponding age- and sex-matched controls (gray circles) are plotted as case-control pairs. B: Scatterplot of FA values in the posterior brainstem compartment. C: The mean FA was significantly higher in the anterior brainstem in patients with CM-I. D: There was a trend toward higher FA in the posterior brainstem in patients with $\mathrm{CM}-\mathrm{I}$, although the difference was not statistically significant. 

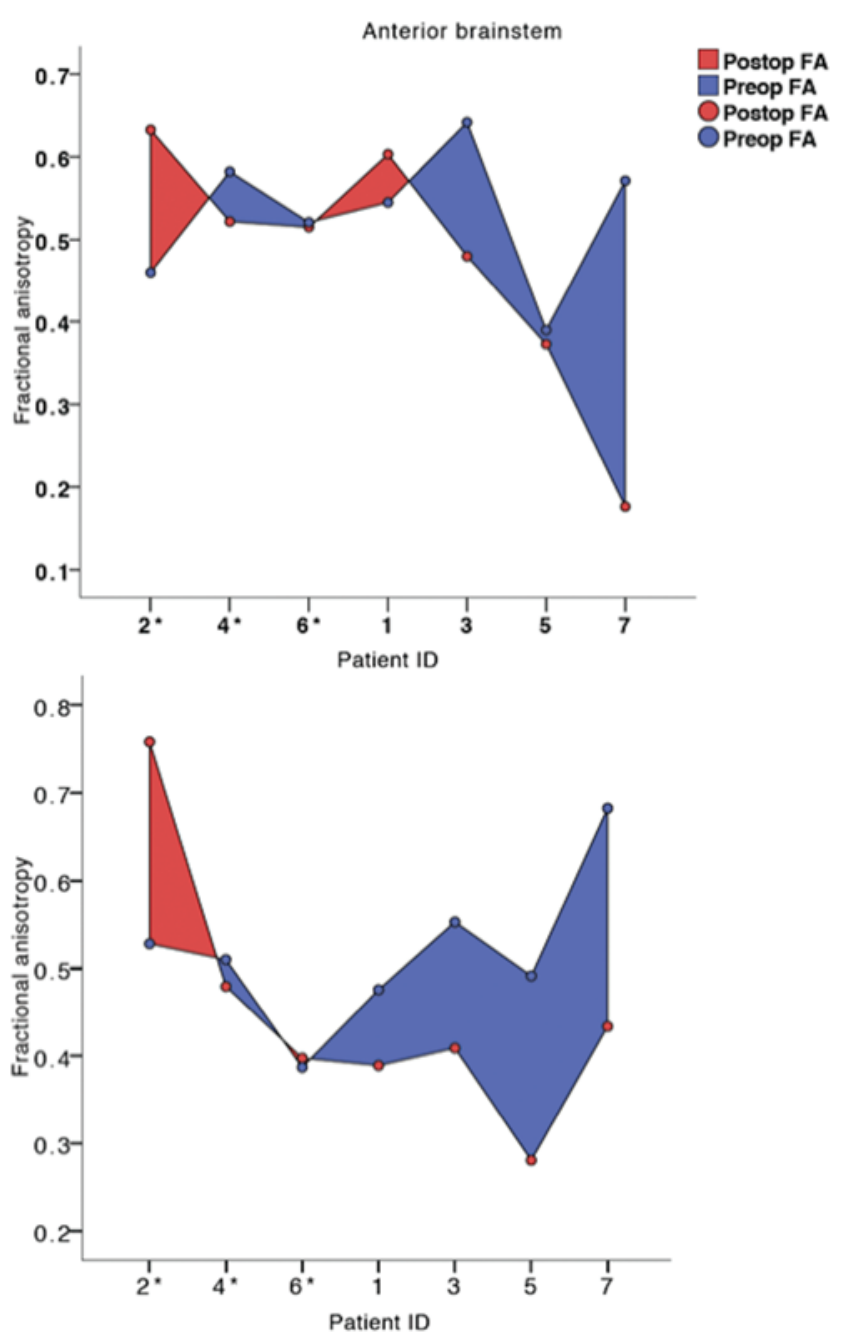

FIG. 3. Area plots of FA values in patients with CM-I at baseline (blue dots) and after surgical decompression (red dots). Red shaded areas signify persistently elevated FA, whereas blue shaded areas denote normalization of FA after surgical decompression. * = Patients with both $\mathrm{CM}-\mathrm{I}$ and syrinx. Figure is available in color online only.

We found that FA was significantly elevated in the anterior brainstem in patients with CM-I. The FA values normalized after decompressive surgery. In patients with syrinx, the FA in the posterior brainstem remained elevated after decompression. These results are in line with our hypothesis that brainstem metrics are clinically more relevant than the degree of tonsillar herniation in the assessment of CM-I.

The diffusion of water molecules is preferential (or anisotropic) in the nervous system, constrained mainly by the tissue microstructure. ${ }^{6,19}$ Diffusion tensor is a common descriptor of diffusion anisotropy. ${ }^{19}$ In this model, the diffusion of water is represented by an ellipsoid with a shape determined by the eigenvalues of the tensor. The diffusivity along the principal axis of this ellipsoid is also termed axial diffusivity, and the average diffusivity along the other 2 minor axes (width and depth) is represented as the radial diffusivity.

Mean diffusivity is another metric to account for the overall diffusion of water molecules, regardless of a spe- cific direction. ${ }^{7}$ Mean diffusivity is thus related to axonal density, myelin integrity, and fiber coherence. However, the voxel-wise anisotropy in diffusion, instead of diffusion per se, correlates with the longitudinal histological changes in the CNS. ${ }^{7}$ The FA relates to the diffusivity in the direction of the largest eigenvalue that is parallel to the main direction of the white matter bundle under investigation. Therefore, the FA is influenced by axonal microstructural integrity and is reliable in regions characterized by a predominant directionality of fiber orientation pattern, e.g., the corpus callosum, cerebral peduncle, and lower brainstem.

We separately calculated the FA values in 2 large regions of interest (ROIs) demarcating the anterior and posterior brainstem compartments. This was performed due to the inherent differences in their functional organization $^{2}$ and the intimate relationship of the posterior brainstem compartment with the fourth ventricle. This makes the posterior brainstem compartment more vulnerable to abnormal CSF dynamics found in patients with CM-I and syrinx. ${ }^{18,23}$

These large brainstem ROIs include both the gray and white matter structures. The anterior brainstem ROI includes the densely packed large white matter tracts (pyramidal tract, medial lemniscus, and central tegmental tract) and inferior olivary nucleus. Similarly, the posterior brainstem contains several gray matter nuclei (hypoglossal nucleus, dorsal motor nucleus of vagus, nucleus tractus solitarius, vestibular nuclei, spinal trigeminal nucleus, and nucleus ambiguous) and a few white matter tracts (medial longitudinal fasciculus, inferior cerebellar peduncle, spinothalamic tract, and spinal trigeminal tract).

The biological basis of anisotropy in the brainstem can help with the interpretation of mean FA values in the brainstem ROIs and the significance of a higher FA in patients with CM-I. The most important determinant of diffusion restriction (hence FA) is the microstructure of the white matter fibers in the nervous system. ${ }^{6,7}$ The axolemma and myelin sheaths surrounding the axons within the laminar organization of white matter tracts are responsible for creating the directionality and restriction in free diffusion of water molecules. ${ }^{6}$ Therefore, despite the inclusion of gray matter nuclei in the ROI, we obtained overall high mean FA values in the brainstem (range 0.42-0.52).

We can assume that a comparison of FA between patients with CM-I and controls reflects the microstructural consequences of brainstem compression. In other words, an elevation of FA in the anterior brainstem, a region with densely packed white matter tracts, may imply a tighter axonal configuration. A similar approach of including both the gray and white matter in FA calculation has previously been published for investigating spinal cord compression ${ }^{11}$ and chronic spinal cord injury. ${ }^{9}$ Indirect support for this explanation comes from the findings of Eshetu et al., who reported a significant decrease in axial diffusivity in the middle cerebellar peduncle of symptomatic patients with CM-I and those without syrinx. ${ }^{10}$ Similarly, others have also reported elevated FA in compressed white matter associated with hydrocephalus ${ }^{5}$ and tumors. ${ }^{26}$

We observed a normalization of FA values in the anterior and posterior brainstem compartments after decompressive surgery. When interpreting the changes in FA 
values, it is important to consider both the absolute value and the direction of change. Because FA is a scalar metric, even small changes may reflect profound microstructural changes associated with various disease processes. Toosy et al. compared the FA values in the corticospinal tract at various levels in patients with amyotrophic lateral sclerosis with those of healthy controls. ${ }^{30}$ They found significantly lower FA at the level of internal capsule, peduncles, pons, and pyramids in patients with amyotrophic lateral sclerosis. The reported difference in magnitude varied from 0.042 to 0.058 .

Similarly, Facon et al. reported the FA changes associated with spinal cord compression. ${ }^{11}$ The mean FA was significantly lower at the site of compression (difference in FA 0.07; $\mathrm{p}=0.12$ ). Nicoletti et al. also reported a difference of 0.1-0.15 in FA in the dentate nucleus and superior cerebellar peduncles in patients with familial essential tremor compared with healthy controls. ${ }^{21}$ Interestingly, in patients with both CM-I and syrinx, the brainstem FA values remained elevated postdecompression. This elevation was not related to an ongoing compression from the syrinx because we observed complete resolution of syrinx in all of the patients (Cases 2, 4, and 6) after decompressive surgery. The significance of elevated FA in patients with syrinx is unclear, although it may signify reorganization following neuronal degeneration in areas surrounding the syrinx.

In contrast to these findings, Abeshaus et al. reported a significant elevation in FA (and a reduction of apparent diffusion coefficient) in the brainstem, cerebellum, and pyramidal tract after decompression in 11 pediatric patients with symptomatic CM-I. ${ }^{1}$ The potential reasons for this difference include our inclusion of the brainstem segment immediately proximal to the site of tonsillar compression, as well as separate analysis of anterior and posterior brainstem compartments.

Overall, FA was better able to distinguish between patients with CM-I and controls compared with the other structural parameters (i.e., posterior fossa volume, total brain volume, fourth ventricular volume, and so on). This is not surprising because the structural parameters are not specific for severity of brainstem compression. Previous studies have also reported poor specificity of structural parameters for ruling out the diagnosis of CM-I..$^{16,25,27}$ At present, we do not advocate the use of an FA threshold for the diagnosis of CM-I. On the other hand, we understand the limitations of relying on a single FA value because these could be affected by a host of variables, including the patient's age and blood pressure. ${ }^{15}$ In fact, in our series, $2(25 \%)$ patients with CM-I had FA values similar to controls.

Serial FA measurements, on the other hand, can be useful for surgical decision making in patients with CM-I. For example, in patients with incidental but significant tonsillar herniation, neurosurgeons often face the dilemma to select patients at highest risk of neurological decline. Similarly, in patients with persistent symptoms after decompressive surgery, an assessment of ongoing brainstem compression can be helpful for deciding between reexploration versus watchful waiting. Clearly, in both these scenarios, conventional imaging does not provide enough information. In theory, the study of brainstem microstructural changes can be a useful adjunct in the neurosurgical assessment of brain imaging in correlation with clinical symptoms. Therefore, future studies to investigate the reproducibility and usefulness of these FA findings are highly desirable.

There are several limitations of our study. Due to a small sample size, we did not analyze the correlation between FA and the severity of clinical symptoms. Also, the segmentation of brainstem into anterior and posterior segments did not allow us to study FA changes in specific brainstem tracts and nuclei. Such analysis is desirable in future studies involving larger patient populations. We excluded 12 patients from the imaging analysis either due to missing images (pre- or postoperative scans) or due to lack of appropriate imaging sequence (e.g., a DTI assay not including the entire length of the brainstem or with significant motion artifact). This major limitation also highlights the challenges associated with obtaining appropriate type of neuroimaging studies to examine the lower brainstem and spinal cord in general.

\section{Conclusions}

In this case-control study, we observed significant elevation of FA in the anterior brainstem in patients with CM-I. These microstructural alterations appear to be more reliable than structural parameters. This may be in line with the common clinical observation that severity of symptoms does not always correlate with the degree of tonsillar herniation. These findings should be verified in future prospective studies with larger sample sizes. Such data could be used to develop guidelines for the assessment of microstructural integrity of brainstem using DTI metrics in the workup of patients with CM-I.

\section{References}

1. Abeshaus S, Friedman S, Poliachik S, Poliakov A, Shaw D, Ojemann JG, et al: Diffusion tensor imaging changes with decompression of Chiari I malformation. Neurosurgery 71:E578, 2012 (Abstract)

2. Afifi AK, Bergman RA: Functional Neuroanatomy: Text and Atlas. New York: McGraw-Hill, 1998

3. Aliaga L, Hekman KE, Yassari R, Straus D, Luther G, Chen $\mathrm{J}$, et al: A novel scoring system for assessing Chiari malformation type I treatment outcomes. Neurosurgery 70:656665, 2012

4. Anderson RC, Emerson RG, Dowling KC, Feldstein NA: Improvement in brainstem auditory evoked potentials after suboccipital decompression in patients with Chiari I malformations. J Neurosurg 98:459-464, 2003

5. Assaf Y, Ben-Sira L, Constantini S, Constantini S, Chang LC, Beni-Adani L: Diffusion tensor imaging in hydrocephalus: initial experience. AJNR Am J Neuroradiol 27:17171724, 2006

6. Beaulieu C: The basis of anisotropic water diffusion in the nervous system - a technical review. NMR Biomed 15:435455, 2002

7. Concha L: A macroscopic view of microstructure: using diffusion-weighted images to infer damage, repair, and plasticity of white matter. Neuroscience 276:14-28, 2014

8. Dyste GN, Menezes AH, VanGilder JC: Symptomatic Chiari malformations. An analysis of presentation, management, and long-term outcome. J Neurosurg 71:159-168, 1989

9. Ellingson BM, Ulmer JL, Kurpad SN, Schmit BD: Diffusion tensor MR imaging in chronic spinal cord injury. AJNR Am J Neuroradiol 29:1976-1982, 2008 
10. Eshetu T, Meoded A, Jallo GI, Carson BS, Huisman TA, Poretti A: Diffusion tensor imaging in pediatric Chiari type I malformation. Dev Med Child Neurol 56:742-748, 2014

11. Facon D, Ozanne A, Fillard P, Lepeintre JF, Tournoux-Facon C, Ducreux D: MR diffusion tensor imaging and fiber tracking in spinal cord compression. AJNR Am J Neuroradiol 26:1587-1594, 2005

12. Greenberg JK, Yarbrough CK, Radmanesh A, Godzik J, Yu M, Jeffe DB, et al: The Chiari Severity Index: a preoperative grading system for Chiari malformation type 1 . Neurosurgery 76:279-285, 2015

13. Kumar M, Rathore RK, Srivastava A, Yadav SK, Behari S, Gupta RK: Correlation of diffusion tensor imaging metrics with neurocognitive function in Chiari I malformation. World Neurosurg 76:189-194, 2011

14. Mac Donald CL, Dikranian K, Bayly P, Holtzman D, Brody D: Diffusion tensor imaging reliably detects experimental traumatic axonal injury and indicates approximate time of injury. J Neurosci 27:11869-11876, 2007

15. Maillard P, Seshadri S, Beiser A, Himali JJ, Au R, Fletcher E, et al: Effects of systolic blood pressure on white-matter integrity in young adults in the Framingham Heart Study: a cross-sectional study. Lancet Neurol 11:1039-1047, 2012

16. Meadows J, Kraut M, Guarnieri M, Haroun RI, Carson BS: Asymptomatic Chiari Type I malformations identified on magnetic resonance imaging. J Neurosurg 92:920-926, 2000

17. Milhorat TH, Chou MW, Trinidad EM, Kula RW, Mandell M, Wolpert C, et al: Chiari I malformation redefined: clinical and radiographic findings for 364 symptomatic patients. Neurosurgery 44:1005-1017, 1999

18. Milhorat TH, Miller JI, Johnson WD, Adler DE, Heger IM: Anatomical basis of syringomyelia occurring with hindbrain lesions. Neurosurgery 32:748-754, 1993

19. Mori S, Zhang J: Principles of diffusion tensor imaging and its applications to basic neuroscience research. Neuron 51:527-539, 2006

20. Nevo U, Hauben E, Yoles E, Agranov E, Akselrod S, Schwartz M, et al: Diffusion anisotropy MRI for quantitative assessment of recovery in injured rat spinal cord. Magn Reson Med 45:1-9, 2001

21. Nicoletti G, Manners D, Novellino F, Condino F, Malucelli E, Barbiroli B, et al: Diffusion tensor MRI changes in cerebellar structures of patients with familial essential tremor. Neurology 74:988-994, 2010

22. Nishikawa M, Sakamoto H, Hakuba A, Nakanishi N, Inoue Y: Pathogenesis of Chiari malformation: a morphometric study of the posterior cranial fossa. J Neurosurg 86:40-47, 1997

23. Oldfield EH, Muraszko K, Shawker TH, Patronas NJ: Pathophysiology of syringomyelia associated with Chiari I malformation of the cerebellar tonsils. Implications for diagnosis and treatment. J Neurosurg 80:3-15, 1994

24. Pollack IF, Pang D, Kocoshis S, Putnam P: Neurogenic dysphagia resulting from Chiari malformations. Neurosurgery 30:709-719, 1992
25. Reich JB, Sierra J, Camp W, Zanzonico P, Deck MD, Plum F: Magnetic resonance imaging measurements and clinical changes accompanying transtentorial and foramen magnum brain herniation. Ann Neurol 33:159-170, 1993

26. Schonberg T, Pianka P, Hendler T, Pasternak O, Assaf Y: Characterization of displaced white matter by brain tumors using combined DTI and fMRI. Neuroimage 30:1100-1111, 2006

27. Sgouros S, Kountouri M, Natarajan K: Posterior fossa volume in children with Chiari malformation Type I. J Neurosurg 105 (2 Suppl):101-106, 2006

28. Stevens EA, Powers AK, Sweasey TA, Tatter SB, Ojemann RG: Simplified harvest of autologous pericranium for duraplasty in Chiari malformation Type I. Technical note. J Neurosurg Spine 11:80-83, 2009

29. Stevens JM, Serva WA, Kendall BE, Valentine AR, Ponsford JR: Chiari malformation in adults: relation of morphological aspects to clinical features and operative outcome. J Neurol Neurosurg Psychiatry 56:1072-1077, 1993

30. Toosy AT, Werring DJ, Orrell RW, Howard RS, King MD, Barker GJ, et al: Diffusion tensor imaging detects corticospinal tract involvement at multiple levels in amyotrophic lateral sclerosis. J Neurol Neurosurg Psychiatry 74:1250-1257, 2003

\section{Disclosures}

The authors report no conflict of interest concerning the materials or methods used in this study or the findings specified in this paper.

\section{Author Contributions}

Conception and design: Hodaie, Krishna, Sammartino. Acquisition of data: Hodaie, Krishna, Yee, Walker, Elias. Analysis and interpretation of data: Hodaie, Krishna, Yee, Walker, Elias. Drafting the article: Krishna, Sammartino. Critically revising the article: Hodaie, Krishna. Reviewed submitted version of manuscript: all authors. Statistical analysis: Krishna. Administrative/technical/material support: Hodaie. Study supervision: Hodaie.

\section{Supplemental Information \\ Previous Presentations}

Portions of this work were presented as a poster at the 83rd Annual Meeting of the American Association of Neurological Surgeons, Washington, DC, May 2-6, 2015.

\section{Correspondence}

Mojgan Hodaie, Division of Neurosurgery, Toronto Western Hospital, 399 Bathurst St., 4W-443, Toronto, ON M5T 2S8, Canada.email: mojgan.hodaie@uhn.ca. 